\title{
Atrial fibrillation and the use of oral bisphosphonates
}

This article was published in the following Dove Press journal:

Therapeutics and Clinical Risk Management

21 March 201 I

Number of times this article has been viewed

\section{Michael Pazianas' \\ Cyrus Cooper ${ }^{1,2}$ \\ Yiting Wang ${ }^{3}$ \\ Jeff L Lange ${ }^{4}$ \\ R Graham G Russell 1,5}

'The Botnar Research Centre, Oxford University, Oxford, UK ${ }^{2}$ MRC Epidemiology Resource Centre, University of Southampton, Southampton, UK; ${ }^{3}$ Warner Chilcott Pharmaceuticals, USA; ${ }^{4}$ Procter \& Gamble Company, Cincinnati, $\mathrm{OH}$, USA; ${ }^{5}$ The Mellanby Centre for Bone Research, Sheffield University, Sheffield, UK
Correspondence: Michael Pazianas The Botnar Research Centre and Oxford University Institute of Musculoskeletal Sciences, Nuffield Department of Orthopaedics, Rheumatology and Musculoskeletal Diseases, Nuffield Orthopaedic

Centre, Headington,

Oxford OX3 7LD, UK

$\mathrm{Tel}+44 I 865737850$

Fax +44I865 227966

Email michael.pazianas@ndorms.ox.ac.uk
Background: Epidemiological studies investigating a possible association between bisphosphonates and atrial fibrillation (AF) have reported conflicting findings. The objective of our study was to determine whether exposure to oral nitrogen-containing bisphosphonates alendronate and risedronate are associated with increased incidence of atrial fibrillation.

Methods: In a retrospective cohort study we analyzed data from three large independent databases, two from the United States (MarketScan ${ }^{\circledR}$ and Ingenix ${ }^{\circledR}$ ) and one from the United Kingdom (THIN). 144,548 women, age 50-89, bisphosphonate users during 2002-2005 were compared to 668,891 sex- and age-matched controls (1:4). Our primary outcome measure was new incident atrial fibrillation for up to three years; Cox models adjusted for disease and drug history were used to estimated relative risks.

Results: We identified a total of 8,001, 1,984, and 817 AF cases in oral bisphosphonate users and nonusers during 744,340 (MarketScan), 243,898 (Ingenix), and 148,779 (THIN) personyears of follow-up, respectively. Compared to nonusers, overall adjusted relative risk (adjRR) (95\% confidence interval $[\mathrm{CI}])$ for $\mathrm{AF}$ in oral bisphosphonates users was 0.92 (0.85-0.99; MarketScan), 1.00 (0.87-1.16; Ingenix), and 0.97 (0.79-1.20; THIN); overall adjRR (95\% CI) for any cardiac dysrrhythmia for MarketScan was 1.01 (0.98-1.05), Ingenix 1.06 (0.99-1.13), and THIN 0.97 (0.79-1.20).

Conclusions: In all three databases from the two countries, the risk of AF or cardiac dysrrhythmia was not increased in postmenopausal women treated for up to three years with oral alendronate or risedronate.

Keywords: atrial fibrillation, chemically induced osteoporosis, drug therapy, alendronate or risedronate, bisphosphonates, adverse effects

\section{Introduction}

Concerns about a possible association of bisphosphonates with events of atrial fibrillation (AF), the most common cardiac arrhythmia, were initially prompted by the HORIZON Pivotal Fracture Trial, ${ }^{1}$ which showed a greater incidence of serious AF adverse events among women treated with once-yearly intravenous zoledronic acid compared to placebo controls. In the similarly designed HORIZON Recurrent Fracture Trial, however, no excess of cardiac arrhythmias, including AF, was seen in patients treated with zoledronic acid. ${ }^{2}$ The oral bisphosphonates alendronate and risedronate are the most commonly used bisphosphonates, primarily prescribed for the prevention or treatment of osteoporosis. ${ }^{3}$ Reanalysis of clinical trial data of alendronate (FIT studies) showed a trend towards an increased risk of serious (though not all) AF events. ${ }^{4}$ However, analysis of adverse cardiovascular events in approxi- 
mately 15,000 postmenopausal women in Phase III clinical trials of risedronate demonstrated no increased risk of AF, classified either as an adverse or serious adverse event, in the risedronate treated vs placebo group. ${ }^{5}$ Similar results have now been reported from a pooled analysis of all four ibandronate pivotal trials. ${ }^{6}$ Furthermore, based on reviews of safety data from placebo-controlled clinical trials, the US Food and Drug Administration announced that no clear association was observed between serious or nonserious AF and overall exposure to bisphosphonates. ${ }^{7}$

Retrospective epidemiological studies on the same issue have reported discordant results. A case control study of about 1,700 women from a state health care delivery system in the US during 2001-2004 showed that ever use of alendronate was associated with an increased risk of AF. ${ }^{8}$ Another population-based study of about 81,700 women in Denmark during 1999-2005 showed that use of oral bisphosphonates (etidronate and alendronate) did not increase the risk of AF. ${ }^{9}$ However, a restricted cohort study from the same country (Denmark) by Abrahamsen et al showed an increased occurrence of AF in fracture patients treated with oral bisphosphonates. ${ }^{10}$ The higher risk of AF in bisphosphonate treated patients, as the authors suggest, could be attributed to their increased baseline risk for cardiovascular events when compared with nonusers. In the United Kingdom (UK), a case-series study showed no overall long-term increased risk of atrial fibrillation with use of alendronate and risedronate. ${ }^{11}$ A meta-analysis by Loke et $\mathrm{al}^{12}$ showed that bisphosphonate use is not associated with a significant increase in the risk of serious AF in postmenopausal women. However, a more recent meta-analysis that included the same studies as Loke's et al, ${ }^{12}$ with the addition of the Abrahamsen et al findings ${ }^{10}$ arrived at a conclusion that an association might exist. ${ }^{13}$ Interestingly, the Abrahamson et al data ${ }^{10}$ included a high proportion of bisphosphonate users compared to controls that had known risk factors for AF at the baseline; thus modulating any impact of the bisphosphonates.

There are no proven mechanisms for this reported association between the use of bisphosphonates and increased incidence of AF. An extensive review of relevant published studies examined the potential relationship between atrial arrhythmogenesis and the pharmacological action of bisphosphonates. ${ }^{14}$ The authors concluded that evidence that bisphosphonates affect atrial conduction is currently lacking but this might be an important area for further investigation.

Observational studies may be well suited for studying associations between commonly used drugs and adverse events. ${ }^{15,16}$ However, bias and confounding is inevitably a practical challenge. More replication studies are needed to scrutinize the association, if any, between oral bisphosphonate use and AF, because both AF and osteoporosis are very common conditions in the elderly. ${ }^{17,18}$ We conducted a retrospective cohort study using data from three large, independent databases in the US and the UK to determine if there was an association between the use of oral bisphosphonates alendronate and risedronate and incidence of AF.

\section{Methods}

\section{Data source}

We obtained data from three large, independent databases: MarketScan $^{\circledR}$, Ingenix ${ }^{\circledR}$, and the Health Improvement Network (THIN).

MarketScan refers to the MarketScan Commercial Claims and Encounters and Medicare Supplemental and Coordinator of Benefits databases. MarketScan obtains administrative data from approximately 45 large employers, health plans, and government and public organizations. Detailed information about the quality of diagnosis, procedure coding and data validation, as well as extensive application of these databases in health care research has been published elsewhere. ${ }^{19}$ As of December 2006, MarketScan represented the medical claims experience of 37 million people, of which 15 million were eligible for a medical claim. The MarketScan data was representative of the age and geographical distribution of the whole US population, with members residing primarily in the South (40\%), as well as in the West (26\%), Midwest (24\%), and Northeast (10\%).

Ingenix refers to Ingenix Lab/Rx Database ${ }^{\circledR}$ (Eden Prairie, $\mathrm{MN})$. Ingenix obtains administrative data from managed-care health plans. As of December 2006, the Ingenix data contained information on 35 million people of which 11 million were currently eligible for a medical claim. Compared to the whole US population, Ingenix data under-represented individuals aged 65 years and over, but covered a wide geographic area, with members residing primarily in the South (45\%) and Midwest (27\%), as well as in the West (15\%) and Northeast (12\%).

Both MarketScan and Ingenix contain longitudinal healthcare utilization data of individual patients, including: 1) inpatient and outpatient clinical services specified by diagnostic codes of the International Classification of Diseases (ICD-9-CM), and procedure (mostly Current Procedural Terminology [CPT] codes); 2) retail and mailorder pharmacy dispensations specified by national drug codes (NDC); and 3) demographic information including age, sex, and dates of eligibility for the respective health 
plan. Both databases record fully adjudicated insurance claims for prescriptions filled by patients, and implement proprietary validation process, however, there is no guarantee that patients actually take the drug.

THIN is a large database of anonymous medical records collected at more than 300 general practices around the UK. ${ }^{20}$ This longitudinal database is regularly updated and contains primary care data of 5.5 million individuals living in the UK. The patient population in THIN is broadly representative of the UK population. THIN is subject to a number of quality control standards to ensure consistent recording of important clinical outcomes and indicators. The quality of the database is monitored, and medical diagnoses in the database have high validity. THIN contains computerized data on patient demographics, medical encounters and diagnosis, prescriptions, and additional health information. Clinical data and diagnoses are cataloged using Read Codes, a comprehensive classification scheme for medical conditions and symptoms. Prescriptions and therapies are entered using Multilex codes, which provide detailed information on the drug, dose, and route of administration.

Claims data of individual patients were de-identified in both Ingenix and MarketScan research databases in compliance with the US Health Insurance Portability and Accountability Act of 1996 (HIPAA). Ethical approval was obtained from the THIN Research Ethics Committee.

\section{Study sample}

In each of the three databases separately, we assembled a cohort of women according to their exposure to bisphosphonates between January 1st, 2002 and December 31st, 2005, as follows (Table 1). Bisphosphonate users included women who were between 50 and 89 years of age when they first received a prescription for alendronate or risedronate during this time period. The date of the first prescription of these drugs for each user was defined as the index date. For each user, up to four nonusers of the same age in years (based on year of birth) on the index date were randomly selected, and assigned the same index date as the user. These age-eligible nonusers were then checked for eligibility based on their medical history before the assigned index date. We decided to execute multiple rounds of this matching and checking process if necessary to avoid excluding more than $1 \%$ of users on account of no matching nonusers, two rounds of matching were done in the MarketScan and Ingenix databases, while one round achieved this in THIN.

To reduce exposure misclassification with respect to subsequent risk of incident $\mathrm{AF}$, we required all women to have at least two years of continuous enrollment before the index date, during which time they must have no bisphosphonate prescriptions and at least one medical encounter. We also excluded women who had any of the following conditions during the two years before their index date:

Table I Creation of study cohorts according to oral bisphosphonate use

\begin{tabular}{|c|c|c|c|c|c|c|}
\hline & \multicolumn{2}{|c|}{ MarketScan (US) ${ }^{a}$} & \multicolumn{2}{|c|}{ Ingenix (US) ${ }^{a}$} & \multicolumn{2}{|c|}{ THIN (UK) } \\
\hline & $\begin{array}{l}\text { Users, } \\
\text { N (\%) }\end{array}$ & $\begin{array}{l}\text { Nonusers, } \\
\mathbf{N}(\%)\end{array}$ & $\begin{array}{l}\text { Users, } \\
\text { N (\%) }\end{array}$ & $\begin{array}{l}\text { Nonusers, } \\
\mathbf{N}(\%)\end{array}$ & $\begin{array}{l}\text { Users, } \\
\text { N (\%) }\end{array}$ & $\begin{array}{l}\text { Nonusers, } \\
\text { N (\%) }\end{array}$ \\
\hline Women aged $50-89$ on index date & 565,370 & $1,232,843$ & 251,680 & 437,362 & 35,744 & 128,950 \\
\hline between $0 I / 0 I / 2002$ and I2/3I/2005 & $(100 \%)$ & $(100 \%)$ & $(100 \%)$ & $(100 \%)$ & $(100 \%)$ & $(100 \%)$ \\
\hline $\begin{array}{l}\text { Above, having at least two years of } \\
\text { continuous enrollment before index date }\end{array}$ & $\begin{array}{l}213,234 \\
(37.7 \%)\end{array}$ & $\begin{array}{l}825,928 \\
(67.0 \%)\end{array}$ & $\begin{array}{l}84,834 \\
(33.7 \%)\end{array}$ & $\begin{array}{l}302,931 \\
(69.3 \%)\end{array}$ & $\begin{array}{l}34,125 \\
(95.5 \%)\end{array}$ & $\begin{array}{l}117,704 \\
(91.3 \%)\end{array}$ \\
\hline $\begin{array}{l}\text { Above, having at least one medical encounter } \\
\text { and no bisphosphonate prescriptions during }\end{array}$ & $\begin{array}{l}167,913 \\
(29.7 \%)\end{array}$ & $\begin{array}{l}769,871 \\
(62.4 \%)\end{array}$ & $\begin{array}{l}64,570 \\
(25.7 \%)\end{array}$ & $\begin{array}{l}269,068 \\
(61.5 \%)\end{array}$ & $\begin{array}{l}28,095 \\
(78.6 \%)\end{array}$ & $\begin{array}{l}113,230 \\
(87.8 \%)\end{array}$ \\
\hline \multicolumn{7}{|l|}{ two years before index date } \\
\hline $\begin{array}{l}\text { Above, having no diagnosis of } \mathrm{AF} \text {, any cardiac } \\
\text { dysrrhythmia, or antiarrhythmic prescriptions } \\
\text { during two years before index date }\end{array}$ & $\begin{array}{l}|5|, 488 \\
(26.8 \%)\end{array}$ & $\begin{array}{l}686,944 \\
(55.7 \%)\end{array}$ & $\begin{array}{l}53,049 \\
(21.1 \%)\end{array}$ & $\begin{array}{l}221,118 \\
(50.6 \%)\end{array}$ & $\begin{array}{l}24,666 \\
(69.0 \%)\end{array}$ & $\begin{array}{l}85,572 \\
(66.4 \%)\end{array}$ \\
\hline $\begin{array}{l}\text { Above, having no diagnosis of hyperthyroidism, } \\
\text { hypothyroidism, alcoholism, or prescriptions } \\
\text { of thyroxin, PTH or strontium during }\end{array}$ & $\begin{array}{l}120,444 \\
(21.3 \%)\end{array}$ & $\begin{array}{l}544,005 \\
(44.1 \%)\end{array}$ & $\begin{array}{l}4 I, 530 \\
(16.5 \%)\end{array}$ & $\begin{array}{l}175,009 \\
(40.0 \%)\end{array}$ & $\begin{array}{l}21,426 \\
(59.9 \%)\end{array}$ & $\begin{array}{l}76,589 \\
(59.4 \%)\end{array}$ \\
\hline \multicolumn{7}{|l|}{ two years before index date } \\
\hline Above, having no oral glucocorticoid & 96,185 & 448,140 & 34,356 & 148,544 & 15,007 & 72,207 \\
\hline prescriptions in two years before index date & $(17.0 \%)$ & $(36.4 \%)$ & $(13.7 \%)$ & $(34.0 \%)$ & $(42.0 \%)$ & $(56.0 \%)$ \\
\hline Above, with up to $4:$ I matching & 95,228 & 352,627 & 33,907 & 122,196 & 14,958 & 54,847 \\
\hline of nonusers to users & $(16.8 \%)$ & $(28.6 \%)$ & $(13.5)$ & $(27.9 \%)$ & $(41.8 \%)$ & $(42.5 \%)$ \\
\hline
\end{tabular}

Note: ${ }^{\mathrm{a}} \mathrm{T}$ wo rounds of matching were used.

Abbreviation: AF, atrial fibrillation. 
supraventricular or ventricular arrhythmia (including pacemaker), hyperthyroidism, hypothyroidism, alcoholism, and prescriptions for antiarrhythmics, thyroxin, strontium, or oral glucocorticoids.

The study cohorts were followed for up to a maximum of three years from the index date until development of any of the study outcomes, ie, AF, any other cardiac dysrrhythmia (including palpitations, bradycardia, tachycardia, etc), prescription of amiodarone, other antiarrhythmic drugs, or were censored for prespecified conditions, ie, death, disenrollment, treatment with oral glucocorticoids, thyroxin, strontium, use of any bisphosphonate (nonusers only), use of a different bisphosphonate than that at the index date (users only, except for switching between alendronate and risedronate in the combined analysis of the two drugs), diagnosis of alcoholism, completion of three years follow up or December 31 st of 2006, whichever came first.

Consistent with previous studies, AF diagnosis included ICD-9 codes 427.31 (AF) and 427.32 (atrial flutter) in the two US databases, and the READ codes 3272.00 (ECG: AF), 3273.00 (ECG: atrial flutter), G573.00 (AF and flutter), G573000 (AF), and G573100 (atrial flutter) in the UK database. We empirically assumed that AF cases identified from hospital settings (including referral) were more severe. We also searched for electrocardiogram (ECG) procedures within one week before or after AF diagnosis, and assumed these cases were additionally supported with ECG evidence.

To explore a more liberal definition of AF based on prescriptions of digoxin, warfarin, or amiodarone, we reviewed complete medical and drug history records available in the database of 100 randomly selected patients (50 users and 50 nonusers). All recorded entries were exported to an Excel spreadsheet, except for bisphosphonate use, osteoporosis, and fracture diagnosis which were maintained in a blinded exposure status.

Our primary event of interest was incident AF. In addition, we also included any other cardiac dysrrhythmia, or prescription of amiodarone as well as AF as part of a composite secondary event of interest. To account for potential confounding factors, we also searched the medical and prescription drug history of the study subjects during the two years before the index date (considered as baseline) for the following conditions: angina, congestive heart failure, diabetes, fractures, any hospitalizations, hypertension, myocardial infarction, osteoporosis, renal diseases, stroke, thrombosis, prescriptions for ACE inhibitors, angiotensin receptor blockers (ARBs), anticoagulants, beta-blockers, calcium channel blockers, medications for cardiac ischemia, estrogens, diuretics, statins, total number of hospitalizations, and total number of all prescriptions.

\section{Data analyses}

Each study cohort from the three different databases was analyzed separately using the same approach outlined below.

Cox models for survival analysis were used to estimate the hazard ratios for AF (primary event of interest) and any dysrrhythmia (secondary event of interest) comparing bisphosphonate users with their matched nonusers. All models were stratified on the matching variables: age and index date, with index date considered as the baseline. Potential confounders included both a prespecified list of potential important confounders based on the literature, and variables automatically selected using stepwise selection from a comprehensive list of variables using available records on inpatient and outpatient medical history, prescription drugs, and procedures in the three databases. Due to the large number of variables and weak assumptions on relevance to confounding, we used the Shtatland et al method ${ }^{21}$ for the stepwise variable selection for both AF and overall dysrrhythmia outcomes separately in the three databases (hence six sets of variables). All variables selected from any one or more of the six sets were kept in final model analysis. Of note, all of our prespecified key confounders were selected by the automatic approach. Proportional hazards assumptions were checked by fitting an interaction term between bisphosphonate use and follow-up time. We also checked that the selected confounders were not highly correlated (ie, multicollinearity).

Primary analysis compared all bisphosphonate users with their matched nonusers in the full study cohort. Secondary analyses were stratified according to 1$)$ age at index date ( $\geq 70$ vs $<70$ years); 2) baseline heart disease (yes vs no), which included any diagnosis of angina, congestive heart failure, myocardial infarction, or other heart diseases; 3) baseline vascular disease and therapy (yes vs no), which included any diagnosis of stroke, thrombosis, or prescriptions for anticoagulants or cardiac ischemic drugs; 4) baseline blood pressure therapy (yes vs no), which included any prescriptions for ACE inhibitors, ARBs, beta blockers, calcium channel blockers, or diuretics; 5) baseline fractures (yes vs no). These conditions were chosen for stratified analysis in order to assess whether any associations between bisphosphonate use and AF may be modified by (ie, depend on, or interact with) age or any major cardiovascular conditions, with somewhat arbitrary groupings that were not meant to be mutually exclusive. 
Furthermore, we:

1. varied the definition of bisphosphonate users as requiring two or more prescriptions of the index drug (index date unchanged), which may reduce misclassification if some patients with only one recorded prescription may not have taken the drug;

2. evaluated whether there was a dose-response relationship in time-dependent Cox models that updated the total cumulative number of bisphosphonate prescriptions monthly during follow-up as: $1,2-10, \geq 11$ prescriptions;

3. divided the follow-up periods into $<90$ days, 90-179 days, 180-364 days, and $\geq 365$ days since index to evaluate both relatively short and long risk windows.

We also separately analyzed alendronate and risedronate users as identified by the index prescription. As a sensitivity analysis, we also tested two different designs of sample selections:

1. same selection of users, but nonusers were randomly selected with a $4: 1$ matching on age to users on $01 / 01 / 2004$, which was the midpoint of the time window for user selection, and assigned to all nonusers;

2. same selection of users, but AF event rates were compared in the three months before and three months after index date among the users only.

All analyses were performed using SAS (v 9.1; SAS Institute, Inc, Cary, NC). Two-sided $P$-values of $<0.05$ from likelihood ratio tests were considered as statistically significant, with no adjustment for multiple comparisons.

\section{Results}

The total number of oral nitrogen containing bisphosphonate users who met all the inclusion criteria in all three databases was calculated to be 144,093 with 529,670 age-matched controls. We identified 1,995 cases of AF (including severe) and 8,057 cases of any cardiac dysrrhythmia in the users group compared to 8,807 cases of AF (including severe) and 32,712 cases of any cardiac dysrrhythmia in the nonusers.

The creation of the study cohorts is illustrated in Table 1. MarketScan was by far the largest database, followed by Ingenix and THIN. While up to one-third of nonusers and two-thirds of users in the two US claims databases were excluded based on less than two years of enrollment before the index date, fewer than $10 \%$ of women in THIN were excluded based on this criterion. Furthermore, only $5 \%$ of the users and $11 \%$ of the nonusers in the two US databases as well as $10 \%$ of the users and $21 \%$ of the nonusers from the THIN database were excluded because of previous diagnosis of AF or other dysrrhythmia.
General descriptions of the three study cohorts according to bisphosphonate use are provided in Table 2. Data available relating to follow-up and age at index date were more enriched in the THIN, followed by MarketScan and Ingenix. AF incidence was largest in MarketScan, followed by Ingenix and THIN. Median follow-up for users was shorter than the matched nonusers in all three databases. Among oral bisphosphonate users in all three databases, the most common oral bisphosphonate regimen was weekly ( $>90 \%)$, and the predominant days supply was for one month $(>80 \%)$ with $<5 \%$ for three months. The reasons for censoring were similar for users and nonusers, with the exception of greater rates of bisphosphonate switching among users (about 10\% in each database) than initiating bisphosphonates among nonusers (about $1 \%$ in each database) during follow-up (data not shown). Overall baseline medical and prescription drug history did not differ by more than $1 \%-2 \%$ between users and nonusers in all three databases, with the exception of osteoporosis ( $>20 \%$ more common among bisphosphonate users than nonusers). In all three databases, there were more nonusers than users with baseline diabetes, hypertension, or use of beta blockers. More bisphosphonate users were taking estrogens while no distinct patterns were noted for the rest of the baseline conditions. Risedronate users were generally older, and had an increased number of baseline cardiovascular diseases and medication use compared with alendronate users with a few exceptions (Table 2).

Overall, the primary analysis (multivariate adjusted models) in all three databases provided no evidence for an association between oral bisphosphonate use and AF in general, severe (hospitalized) cases alone, or ECG supported cases only. Furthermore, no association was detected when all dysrrhythmias were analyzed together. The overall hazard ratio for AF was 0.93 (0.88-0.99), $P=0.02$. Oral bisphosphonate use was associated with an adjusted $8 \%$ lower AF risk in the MarketScan database. Figure 1A and 1B illustrate the Kaplan-Meier estimates of the cumulative incidence curves for AF and dysrrhythmia, respectively. Hazard ratio estimates from all Cox models for AF in general, severe (hospitalized) cases or ECG supported cases, and dysrrhythmia are shown in Tables 3, 4, and 5, respectively. Variance inflation factors for all selected confounders were low $(<2)$. In the two US databases, hazard ratio estimates for bisphosphonate use in patients older than 70 years of age appeared similar to estimates in younger patients. Furthermore, the hazard ratio estimates did not change when we compared bisphosphonate users to nonusers and the analysis was restricted to patients of 80 years of age or older at the index date (Table 6). 


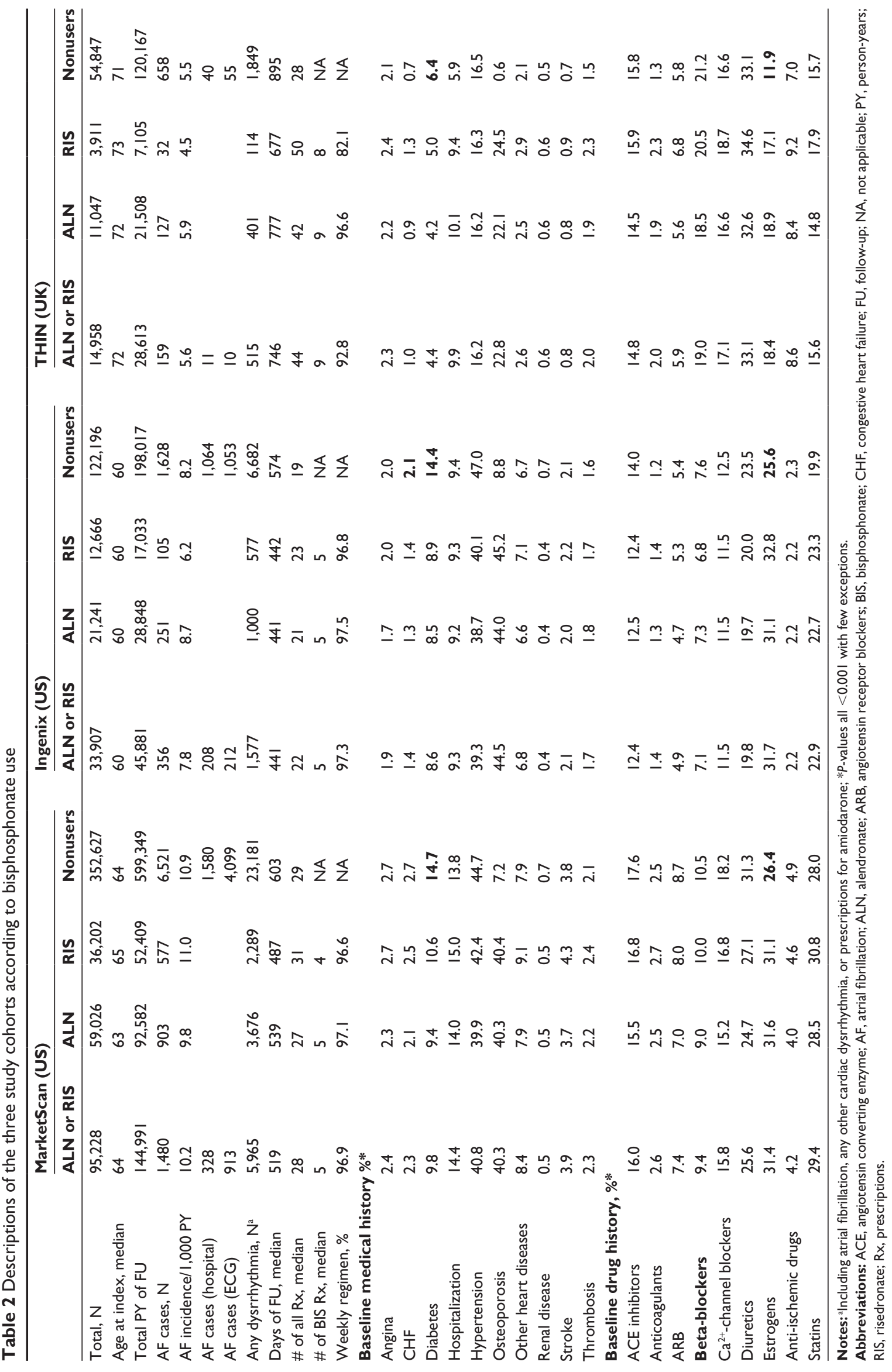




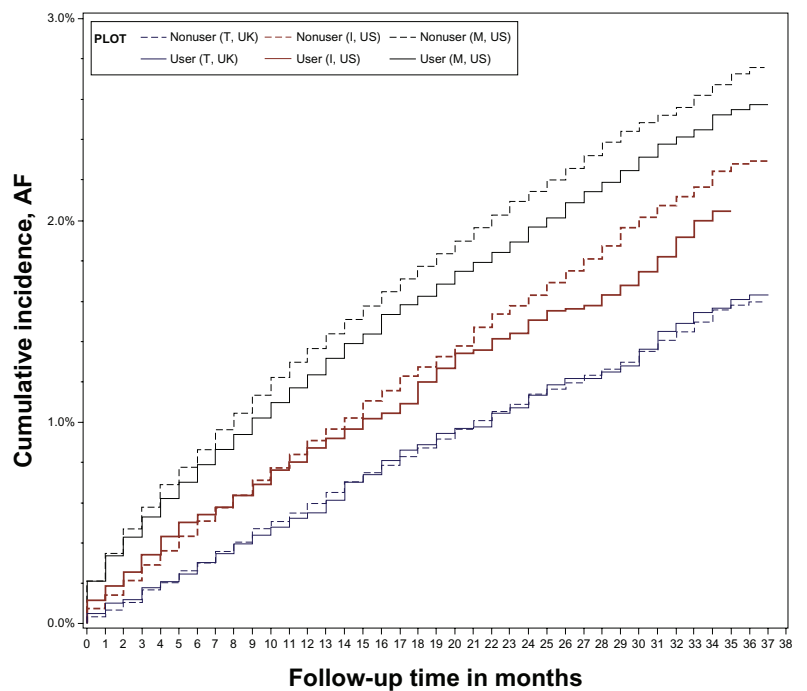

Figure IA Graphical presentation of the overall cumulative incidence of AF, unadjusted for any covariates.

Abbreviations: AF, atrial fibrillation; I, Ingenix; M, MarketScan; T, THIN.

Multivariate models confirmed that the risk factors for AF identified in our population are in agreement with the established ones. Stratified analysis showed that AF risk, independently of oral nitrogen containing-bisphosphonate use, was generally higher among women with known AF risk factors, including older age, cardiovascular diseases, and therapy. Interestingly, anticoagulants came up as the strongest risk factor. The absolute magnitude of the hazard ratio associated with ever use vs never use of anticoagulants at baseline was the largest (data not shown). Also, significant interactions were detected between bisphosphonate use and vascular disease in MarketScan and Ingenix, between bisphosphonate

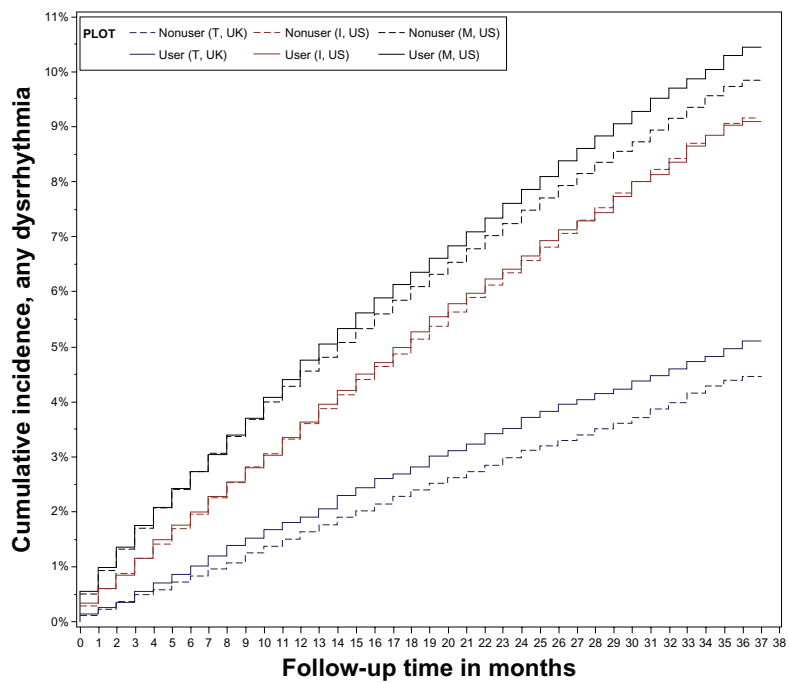

Figure IB Graphical presentation of the overall cumulative incidence for all dysrrhythmias, unadjusted for any covariates.

Abbreviations: AF, atrial fibrillation; I, Ingenix; M, MarketScan; T, THIN. use and blood pressure therapies in MarketScan (Table 3) but no statistical interaction for dysrrhythmia was detected. Statin use (yes vs no at baseline) showed a consistent favorable effect (hazard ratios about 0.8 ) in the present study, although we did not study men and did not measure duration or compliance.

When follow-up was broken up into four intervals, hazard ratios appeared to decrease overall with time since index date, although they did not exhibit well-ordered patterns in MarketScan and THIN. Dose-response analysis showed that each additional oral bisphosphonate prescription was significantly associated with a lower AF risk in Ingenix, but not in MarketScan and THIN. Sensitivity analysis using alternative study designs described in the methods section did not show significantly increased risk with oral bisphosphonate use. Corresponding results for all dysrrhythmias appeared similar, but corresponding hazard ratio estimates were generally larger.

\section{Discussion}

In our study, the biggest retrospective cohort study so far, the estimated incidence of AF was not increased in users of the oral bisphosphonates, alendronate or risedronate. Additionally, the risk factors for AF were no different from those already established, further supporting our conclusion. Equally pertinent is our observation that the mean age at which AF was diagnosed is the same for oral nitrogen containing bisphosphonate users as for nonusers. If bisphosphonate usage does in fact increase the risk of AF, we may expect to see a shift towards a younger age in the users group. Our study also shows no increased incidence of severe AF (hospitalized or ECG supported cases) in oral bisphosphonates users compared with nonusers. In contrast, in the HORIZON study ${ }^{1}$ where IV zoledronate was used, a statistically significant increase in severe AF was observed. Therefore, if indeed there is any real possibility that nitrogen containing bisphosphonates could cause $\mathrm{AF}$, then, oral preparations should be placed at the lowest possible level of risk. Interestingly, combined analysis of all three databases showed that use of oral bisphosphonates was associated with a statistically significant risk reduction in AF. It is beyond the scope of the present study to investigate whether osteoporosis treatment actually results in cardiovascular benefits, and whether such benefits are clinically significant.

In all three databases, we found no increased risk of AF with use of oral bisphosphonates (alendronate and risedronate), consistent with the largest case-control study of Sorensen et al, ${ }^{9}$ the UK case series study using the General 


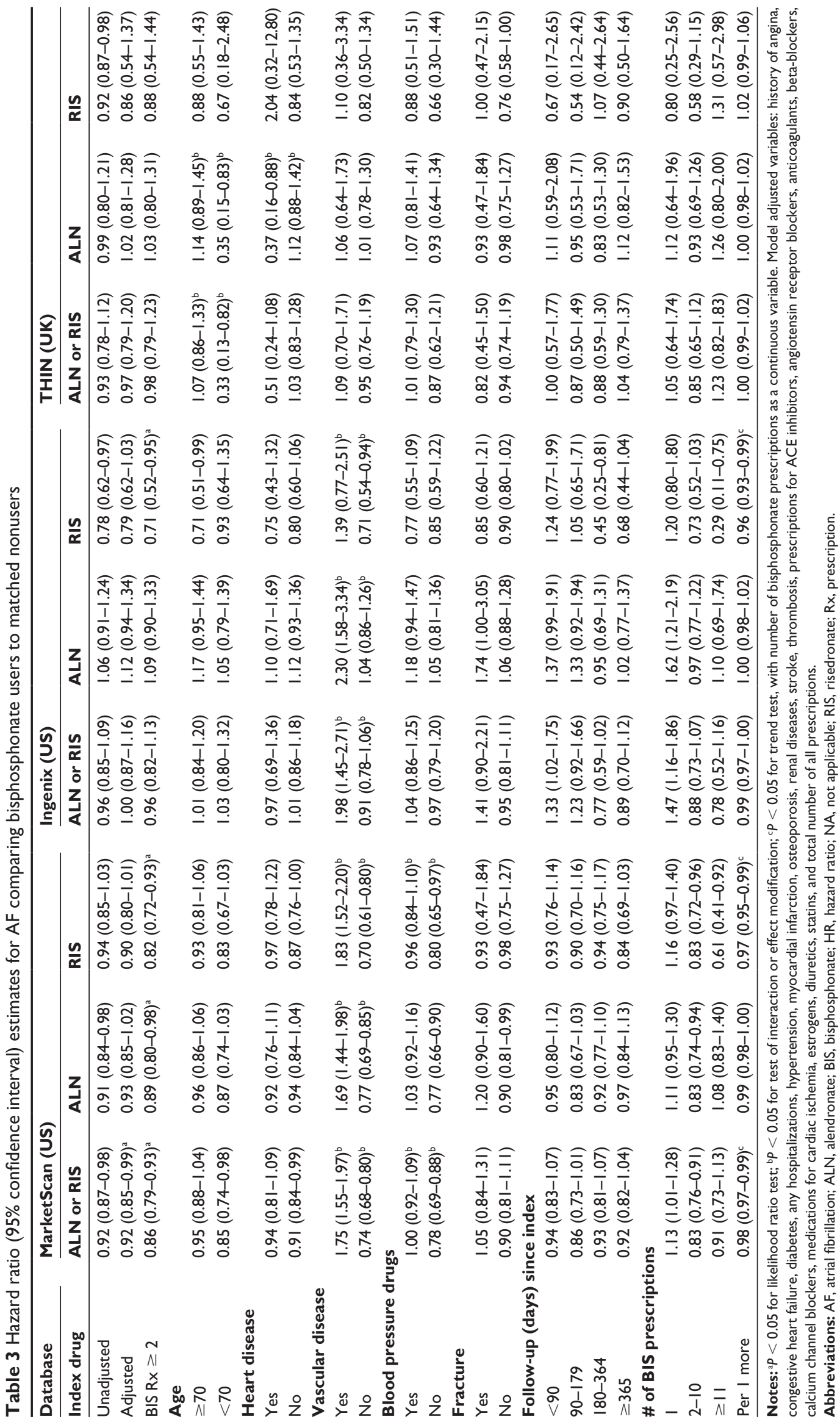


Table 4 Hazard ratio ( $95 \%$ confidence interval) for a) hospitalized AF cases and b) ECG confirmed AF cases

\begin{tabular}{lll}
\hline MarketScan & Ingenix & THIN \\
\hline a) & $0.96(0.80-1.16)$ & $0.96(0.37-2.5 I)$ \\
$0.89(0.77-1.03)$ & $0.94(0.78-1.13)$ & $0.64(0.28-1.46)$ \\
b)
\end{tabular}

Abbreviations: AF, atrial fibrillation; ECG, electrocardiogram.

Practice Research Database (GPRD), ${ }_{11}^{1}$ and published data on a placebo-controlled clinical trial of risedronate for the treatment of osteoporosis..$^{5}$ In contrast, Heckbert et al ${ }^{8}$ reported an overall increased risk of $\mathrm{AF}$ associated with alendronate. Cases in that study were four years older than controls in median age, and had significantly more prevalent AF risk factors. In fact, our stratified analyses showed a general tendency toward larger hazard ratios among women who were older or had cardiovascular risk factors. It is possible that women with baseline stroke, thrombosis, or prescriptions for anticoagulants or cardiac ischemic drugs had prevalent undiagnosed AF. Among women who were younger or had no baseline cardiovascular risk factors, however, the hazard ratio estimates which were not confounded by these risk factors were consistently lower.

We identified ECG supported AF diagnosis as well as cases identified in hospital settings. The results of Cox models on these presumably more specific AF outcomes were very similar to the primary analysis. In addition, the relative risks estimated for established risk factors were generally consistent in magnitude with those reported in previous studies. The incidence rates of $\mathrm{AF}$ diagnosis seemed higher in the two US databases than THIN, even though THIN patients were older. Although there have been reports of increasing trend of AF incidence in the US, ${ }^{17,22}$ we cannot confirm if the difference we observed between the two countries is real. The fact that more prevalent AF cases were excluded in THIN than in the two US databases may in part explain the difference, and it is possible that some AF cases in the US databases were actually prevalent or silent AF cases instead of incident ones. On the other hand, the prevalence of sustained silent AF in people over the age of 65 is believed to be $25 \%-30 \%$ or even higher, ${ }^{23}$ and therefore, both our study and previous ones may have missed many incident cases and included prevalent cases.

We found no increased hazard ratio for AF or cardiac dysrrhythmias in bisphosphonates users older than 70 years of age (Tables 3 and 5). Even when we restricted our comparison of bisphosphonate users with nonusers to patients who were 80 years of age or older, the hazard ratio did not become significant (Table 6). However, our sample size and statistical power was limited in this age group.

Hazard ratio estimates for AF and dysrrhythmia suggested a significant interaction between bisphosphonate use and vascular disease in MarketScan and Ingenix databases (Tables 3 and 5). This finding could well be related to the identification of anticoagulants as the strongest risk factor for AF in both groups, ie, users and nonbisphosphonate users. Also, a significant interaction was detected between bisphosphonate use and blood pressure therapies, albeit in MarketScan database only. It is interesting that in nonbisphosphonate users in all three databases, such blood pressure medications have been implicated as risk factors for $\mathrm{AF}$ and cardiac dysrrhythmias as well. This is contrary to the current understanding that, for example, ACE inhibitors/ARBs have a protective effect.

It is of interest to evaluate whether the initiation of bisphosphonates triggers a transient increase in the risk of AF, which appeared higher following one prescription of a bisphosphonate, even though overall dose-response relationship appeared negative in our study. Neither of the two sensitivity analyses in a short risk window (follow-up $<90$ days, and risk in three months before and after bisphosphonate use) found any significant evidence for increased AF incidence among bisphosphonate users. From the time-dependent Cox model, women who had one prescription only may experience AF long after the prescription date (eg, one year later). These patients may have higher risk of $\mathrm{AF}$ due to poor general health and noncompliance with their therapy. In a sensitivity analysis (not shown) that excluded women with one prescription only, the corresponding HR estimates in time-dependent Cox models associated with one prescription were all diminished. Another reason for caution is that in electronic databases, filling a prescription (the two US databases) or receiving a prescription (UK database) is not equivalent to patients actually taking the drug. As shown in a subanalysis of users with two or more prescriptions of bisphosphonates and their matched nonusers (Tables 3 and 5), hazard ratio estimates decreased after excluding the one prescription only users. The initiation of bisphosphonate therapy may arise from increasing health care encounters and result in early detection and diagnosis of AF during checkup, which may also help to explain the apparent increased risk of AF following one prescription. In the HORIZON study, AF events did not cluster immediately after zoledronic acid infusion, but rather occurred evenly over the three-year period of the study. ${ }^{1,24,25}$ In our study, the time to AF onset 


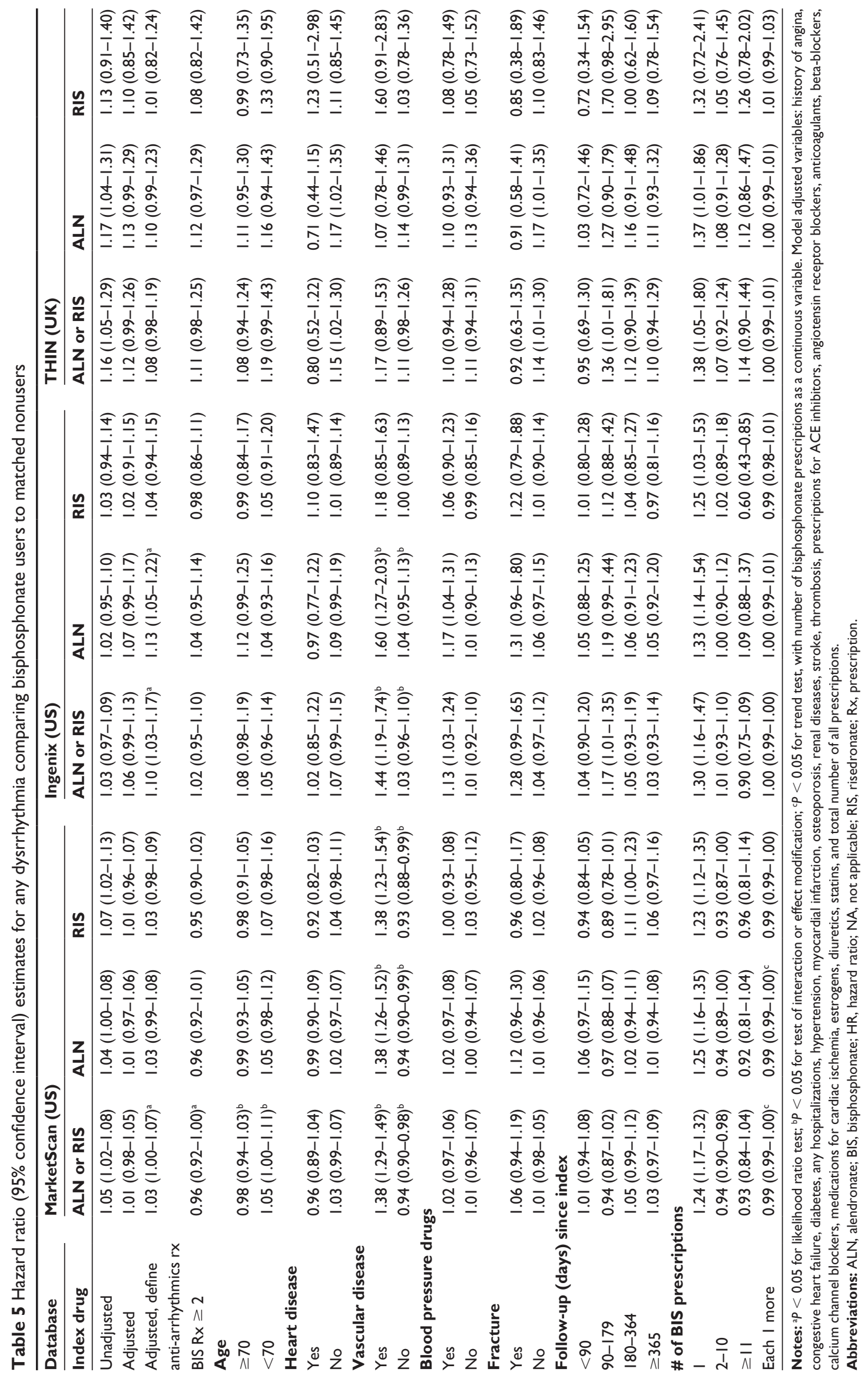


Table 6 Hazard ratio (95\% confidence interval) estimates comparing bisphosphonate users (alendronate and risedronate) to nonusers, restricted to patients of 80 years of age or older at the index date

\begin{tabular}{lllll}
\hline & P-value & $\begin{array}{l}\text { Hazard } \\
\text { ratio }\end{array}$ & Lower & Upper \\
\hline MarketScan, AF & 0.10 & 1.10 & 0.98 & 1.25 \\
Ingenix, AF & 0.69 & 1.05 & 0.82 & 1.35 \\
THIN, AF & 0.81 & 0.96 & 0.72 & 1.29 \\
MarketScan, & 0.42 & 1.03 & 0.96 & 1.10 \\
all dysrrhythmias & & & & \\
Ingenix, all dysrrhythmia & 0.13 & 1.12 & 0.97 & $1.3 \mathrm{I}$ \\
THIN, all dysrrhythmia & 0.21 & 1.15 & 0.93 & 1.42 \\
\hline
\end{tabular}

Abbreviation: $\mathrm{AF}$, atrial fibrillation.

was not evenly distributed in all three databases and AF onset occurred later in time. Among bisphosphonate users, about $27 \%, 23 \%$, and $11 \%$ of AF occurred within first three months of the index date, $35 \%, 37 \%$, and $57 \%$ after one year after the index date in the MarketScan, Ingenix, and THIN databases, respectively. When we divided follow-up into four intervals, only the Ingenix data suggested an increased risk of AF shortly after initiation of bisphosphonate therapy. Further analysis showed that the short-term increased risk of AF mostly occurred in bisphosphonate users who received only one prescription. The baseline cardiovascular risk factors were more prevalent among these patients. Overall data from all three databases did not suggest oral bisphosphonates as an acute trigger for AF events. It is interesting that although risedronate users in all three databases were slightly older than alendronate users, and had in general more cardiovascular risk factors, use of risedronate did not show an increased risk shortly after index date, except in the Ingenix database.

In previous studies, no dose-response relationship was observed for alendronate ${ }^{8}$ or zoledronic acid ${ }^{1}$ in association with AF. In our dose-response analysis, we actually observed a significantly lower risk of AF with more use of bisphosphonates in the two US databases. The Danish case-control study and the present study also did not show any trends toward greater risk of AF with larger cumulative dosing or more recent use of oral bisphosphonates. Paradoxically, stratified analysis in the Heckbert et al study showed that past but not current use of alendronate was associated with AF, with a median time since the last alendronate prescription of 1.3 years among cases and 0.8 years among controls. ${ }^{8}$

Inflammatory events are also associated with $\mathrm{AF}^{26}$ and increased levels of inflammatory markers have been observed both in the acute-phase responses to bisphosphonates ${ }^{27,28}$ and AF. $^{29}$ Treatment with potent anti-inflammatory medications could modify the risk of arrhythmias and thus the outcome of studies on AF. We assessed the effects of statins, a group of agents with anti-inflammatory properties and potentially preventive effects in the development of AF. Our study supports a protective role for statins whereas the Heckbert et al study showed higher risk of AF in patients currently taking statins. ${ }^{8}$

Glucocorticoids, another group of potent anti-inflammatory medications, reduce the release of several inflammatory and acute-phase response mediators but their use is associated with an increased risk of myocardial infarction, stroke, and heart failure, which are also risk factors for AF. A recent report suggested that current use of systemic glucocorticoids is associated with an almost twofold increased risk of AF or flutter. ${ }^{30}$ Interestingly, a significant number of patients on glucocorticoids are treated with an oral bisphosphonate for the prevention or treatment of osteoporosis. In our study we excluded patients on glucocorticoid treatment, especially as in our initial selection of patients twice as many of the bisphosphonate users were taking oral glucocorticoids compared to controls (Table 1).

\section{Strengths and limitations of study}

The strengths of our study include the large sample size (larger than the four studies published so far), application of the same methods to three independent databases of two developed countries, and control of important confounders, ie, age and gender. Studying the three databases separately enabled us to examine the reproducibility of findings in our own study as well as in other previous studies. Consistency of results across different populations and studies is an important consideration in epidemiology. ${ }^{32}$ Because of different health care systems and data structure of the three databases, keeping them separate helps avoid any particular information bias in one database to be repeated in the others. We note that some apparent differences in prevalence of comorbidities between the two countries (eg, osteoporosis, angina, CHF, diabetes) may at least in part due to underascertainment or underrecording of the conditions.

Our study period of 2002-2006 overlaps with those of previous observational studies, ie, before the publication of the HORIZON clinical trial, and thus, is not likely to be subject to greater reporting of AF among users. On the other hand, this calendar time constraint combined with a high turnover rate in our study (especially the two claims databases) limited our ability to extrapolate the results to more than three years of bisphosphonate therapy.

Our study also has other limitations, including lack of sufficient clinical details to confirm AF outcomes or to 
control for disease severity of osteoporosis and cardiovascular disease. It is also possible that prevalent AF and other disease conditions were not fully captured in the two years of history before index. The impact of such measurement bias may be small.

Confounding by indication may not be adequately controlled. Osteoporosis is a risk factor for AF. Bisphosphonates are indicated for the treatment of osteoporosis. Thus therapy with bisphosphonates may be associated with incidence of AF. Osteoporosis has been shown to be a risk factor for incident cardiovascular events, independent of age, diabetes, hypertension, hyperlipidemia, smoking, and prior CHD events. ${ }^{16,24,31}$ Therefore bisphosphonate therapy may be statistically associated with adverse cardiovascular outcomes through its indication for osteoporosis alone. To alleviate such confounding by indication, it is important to assess the specificity of any associations. For example, we explored the specificity of AF case definition based on prescription of warfarin and digoxin. Blinded review with respect to bisphosphonate exposure ruled out 44 (88\%) of the 50 randomly selected nonusers and 40 (90\%) of the 50 randomly selected users as likely to be other cardiovascular events instead of AF. Furthermore, we evaluated a composite outcome defined by any cardiac dysrrthymia diagnosis or antiarrhythmic prescriptions (listed in Appendix 1), the number of cases so defined increased fivefold of that for AF cases, and hazard ratio estimates for bisphosphonate use overall were slightly larger (results not shown). Such a composite outcome likely represented diverse cardiovascular disorders. One of the most commonly used criteria to judge causality involves specificity in the association between a risk factor and outcome. If bisphosphonates truly caused AF, we would generally expect to see a less distinctive association when outcomes became less specific.

We note that in all three databases, a greater percentage of nonusers either had a full three-year follow-up or reached the end of the study (ie, administrative censoring) (54\%, 33\%, and 77\% in MarketScan, Ingenix and THIN, respectively) than users $(39 \%, 25 \%$, and $61 \%$ in MarketScan, Ingenix, and THIN, respectively), and that users were more likely to encounter the censoring events such as switching therapy, receiving glucocorticoid therapy, parathyroid hormone, or thyroxine which may alter the risk of AF (independent of bisphosphonates). Although we didn't directly assess the impact of this censoring on our primary analysis, sensitivity analysis using alternative design yielded similar results. Technically, the numerical magnitude of the longer follow-up in nonusers than users may not have as much real impact on the Cox models, which rely on the ranking of event times rather than the actual length of follow-up. Finally, our study is an observational study and therefore causality cannot be assumed. Furthermore, our findings cannot be extrapolated to male patients or patients older than 90 years of age.

In conclusion, we found that the use of oral bisphosphonates was not associated with an increased incidence of AF. The considerable size of the populations we studied derived from two different countries increases our confidence that our findings reflect the clinical reality in medical practice.

\section{Conflict of interest statement}

M Pazianas has received a grant/research support from Procter and Gamble and Sanofi-Aventis through the University of Oxford by The Alliance for Better Bone Health; C Cooper has received honoraria and/or consulting fees from The Alliance for Better Bone Health, MSD, Eli Lilly Ltd, Novartis, and Amgen Pharmaceutical companies; Y Wang is an employee of Warner Chilcott Pharmaceuticals; J Lange is an employee of Procter and Gamble Company; G Russell is a consultant/ advisor to Amgen, Glaxo-Smith-Kline, Eli Lilly, Novartis, Roche, Procter and Gamble, Sanofi-Aventis, and Warner Chilcott Pharmaceuticals.

\section{References}

1. Black DM, Delmas PD, Eastell R, et al; HORIZON Pivotal Fracture Trial Once-yearly zoledronic acid for treatment of postmenopausal osteoporosis. N Engl J Med. 2007;356:1809-1822.

2. Lyles KW, Colón-Emeric CS, Magaziner JS, et al; for the HORIZON Recurrent Fracture Trial Zoledronic Acid in Reducing Clinical Fracture and Mortality after Hip Fracture. $N$ Engl $J$ Med. 2007;357:1799-1809.

3. Pazianas M, Cooper C, Ebetino FH, Russell RG. Long-term treatment with bisphosphonates and their safety in postmenopausal osteoporosis. Ther Clin Risk Manag. 2010;6:325-343.

4. Cummings SR, Schwartz AV, Black DM. Alendronate and atrial fibrillation. N Engl J Med. 2007;356:1895-1896.

5. Karam R, Camm J, McClung M. Yearly zoledronic acid in postmenopausal osteoporosis. N Engl J Med. 2007;357:712-713.

6. Lewiecki EM, Cooper C, Thompson E, Hartl F, Mehta D, Papapoulos SE. Ibandronate does not increase risk of atrial fibrillation in analysis of pivotal clinical trials. Int J Clin Pract. 2010;64:821-826. Epub 2010 Mar 11.

7. Food and Drug Administration (FDA). The FDA safety information and adverse event reporting program. Available at: http://www.fda. gov/medwatch/safety/2008/safety08.htm\#bisphosphonates2. Accessed February 17, 2011.

8. Heckbert SR, Li G, Cummings SR, Smith NL, Psaty BM. Use of alendronate and risk of incident atrial fibrillation in women. Arch Intern Med. 2008;168:826-831.

9. Sørensen HT, Christensen S, Mehnert F, et al. Use of bisphosphonates among women and risk of atrial fibrillation and flutter: population based case-control study. BMJ. 2008;336:813-816.

10. Abrahamsen B, Eiken P, Brixen K. Atrial fibrillation in fracture patients treated with oral bisphosphonates. $J$ Intern Med. 2009;265: 581-592. 
11. Grosso A, Douglas I, Hingorani A, MacAllister R, Smeeth L. Oral bisphosphonates and risk of atrial fibrillation and flutter in women: a self-controlled case-series safety analysis. PLOS ONE. 2009;4:e4720. Epub 2009 Mar 6.

12. Loke YK, Jeevanantham V, Singh S. Bisphosphonates and atrial fibrillation: systematic review and meta-analysis. Drug Saf. 2009; 32:219-228.

13. Bhuriya R, Singh M, Molnar J, Arora R, Khosla S. Bisphosphonate use in women and the risk of atrial fibrillation: a systematic review and meta-analysis. Int J Cardiol. 2010;142:213-217. Epub 2010 Jan 3.

14. Pazianas M, Compston J, Huang CL. Atrial fibrillation and bisphosphonate therapy. J Bone Miner Res. 2010;25:2-10.

15. Vandenbroucke JP. When are observational studies as credible as 11 randomised trials? Lancet. 2004;363:1728-1731.

16. Majumdar SR. Oral bisphosphonates and atrial fibrillation. BMJ. 2008;336:784-785. Epub 2008 Mar 11.

17. Kannel WB, Wolf PA, Benjamin EJ, Levy D. Prevalence, incidence, prognosis, and predisposing conditions for atrial fibrillation: populationbased estimates. Am J Cardiol. 1998;82(8A):2N-9N.

18. Ruigómez A, Johansson S, Wallander MA, Rodríguez LA. Incidence of chronic atrial fibrillation in general practice and its treatment pattern. J Clin Epidemiol. 2002;55:358-363.

19. Adamson DM, Chang S, Hansen LG. Health research data for the real world: the Marketscan databases. New York: Thompson Healthcare 2008.

20. Lewis JD, Schinnar R, Bilker WB, Wang X, Strom BL. Validation studies of the health improvement network (THIN) database for pharmacoepidemiology research. Pharmacoepidemiol Drug Saf. 2007;16:393-401.

21. Shtatland ES, Kleinman K, Cain EM. Model building in Proc Phreg with automatic variable selection and information criteria. Online proceedings paper, SAS Users Global Forum 2005. Available at: http://www2.sas com/proceedings/sugi30/206-30.pdf. Accessed February 17, 2011

22. Miyasaka Y, Barnes ME, Gersh BJ, et al. Secular trends in incidence of atrial fibrillation in Olmsted County, Minnesota, 1980 to 2000, and implications on the projections for future prevalence. Circulation. 2006 Jul 11;114:119-125. Epub 2006 Jul 3. Erratum in: Circulation. 2006;114:e498.
23. Savelieva I, Camm AJ. Clinical relevance of silent atrial fibrillation: prevalence, prognosis, quality of life, and management. J Interv Card Electrophysiol. 2000;4:369-382.

24. Black DM, Delmas PD, Eastell R; HORIZON Pivotal Fracture Trial. Once-yearly zoledronic acid for treatment of postmenopausal osteoporosis. N Engl J Med. 2007;356:1809-1822. Comment in 2007;35:713; author reply 714-715.

25. Cauley JA, Ensrud KE. Considering competing risks ... Not all black and white. Arch Intern Med. 2008;168:793-795.

26. Boos CJ, Lip GY. Inflammation and atrial fibrillation: cause or effect? Heart. 2008;94:133-134.

27. Santini D, Fratto ME, Vincenzi B, La Cesa A, Dianzani C, Tonini G. Bisphosphonate effects in cancer and inflammatory diseases: in vitro and in vivo modulation of cytokine activities. BioDrugs. 2004; 18:269-278

28. Hewitt RE, Lissina A, Green AE, Slay ES, Price DA, Sewell AK. The bisphosphonate acute phase response: rapid and copious production of proinflammatory cytokines by peripheral blood gd T cells in response to aminobisphosphonates is inhibited by statins. Clin Exp Immunol. 2005;139:101-111.

29. Aviles RJ, Martin DO, Apperson-Hansen C, et al. Inflammation as a risk factor for atrial fibrillation. Circulation. 2003;108:3006-3010.

30. Christiansen CF, Christensen S, Mehnert F, Cummings SR, Chapurlat RD, Sørensen HT. Glucocorticoid use and risk of atrial fibrillation or flutter: a population-based, case-control study. Arch Intern Med. 2009;169:1677-1683.

31. Perrio M, Voss S, Shakir SA. Application of the bradford hill criteria to assess the causality of cisapride-induced arrhythmia: a model for assessing causal association in pharmacovigilance. Drug Saf. 2007; 30:333-346.

32. Tanko LB, Christiansen C, Cox DA, Geiger MJ, McNabb MA, Cummings SR. Relationship between osteoporosis and cardiovascular disease in postmenopausal women. J Bone Miner Res. 2005;20: 1912-1920. 


\section{Appendix I}

Antiarrhythmics (including various formulations or compounding)

Adenosine, atropine, bretylium tosylate, disopyramide, dofetilide, encainide, flecainide, ibutilide, lidocaine, lignocaine, mexiletine, moracizine, procainamide, propranolol, quinidine, sotalol, tocainide, verapamil.

\section{Publish your work in this journal}

Therapeutics and Clinical Risk Management is an international, peerreviewed journal of clinical therapeutics and risk management, focusing on concise rapid reporting of clinical studies in all therapeutic areas, outcomes, safety, and programs for the effective, safe, and sustained use of medicines. This journal is indexed on PubMed Central, CAS,
EMBase, Scopus and the Elsevier Bibliographic databases. The manuscript management system is completely online and includes a very quick and fair peer-review system, which is all easy to use. Visit http://www.dovepress.com/testimonials.php to read real quotes from published authors. 